Pacific Journal of Mathematics

ON THE INVERSE FUNCTION THEOREM 


\title{
ON THE INVERSE FUNCTION THEOREM
}

\author{
F. H. Clarke
}

The classical inverse function theorem gives conditions under which a $C^{r}$ function admits (locally) a $C^{r}$ inverse. The purpose of this article is to give conditions under which a Lipschitzian (not necessarily differentiable) function admits (locally) a Lipschitzian inverse. The classical result is a special case of the theorem.

1. Introduction. Let $f: R^{n} \rightarrow R^{n}$ satisfy a Lipschitz condition in a neighborhood of a point $x_{0}$ in $R^{n}$. Thus for some constant $K$, for all $x$ and $y$ near $x_{0}$, we have

$$
|f(x)-f(y)| \leqq K|x-y|
$$

where $|\cdot|$ denotes the usual Euclidean norm. The usual $n \times n$ Jacobian matrix of partial derivatives, when it exists, is denoted $J f(x)$. We topologize the vector space $M$ of $n \times n$ matrices with the norm

$$
\|M\|=\max \left|m_{i j}\right|
$$

where

$$
M=\left(m_{i j}\right), \quad 1 \leqq i \leqq n, \quad 1 \leqq j \leqq n .
$$

Definition 1. The generalized Jacobian of $f$ at $x_{0}$, denoted $\partial f\left(x_{0}\right)$, is the convex hull of all matrices $M$ of the form

$$
M=\lim _{\imath \rightarrow \infty} J f\left(x_{\imath}\right),
$$

where $x_{i}$ converges to $x_{0}$ and $f$ is differentiable at $x_{t}$ for each $i$.

The above extends to vector-valued functions the notion of "generalized gradient" introduced by the author in [2]. It is a consequence of Rademacher's theorem that $f$ is almost everywhere differentiable near $x_{0}$. Furthermore, $J f(x)$ is bounded near $x_{0}$ as a result of (1). These observations imply

Proposition 1. $\partial f\left(x_{0}\right)$ is a nonempty compact convex subset of $\mathcal{M}$.

Definition 2. $\partial f\left(x_{0}\right)$ is said to be of maximal rank if every $M$ in $\partial f\left(x_{0}\right)$ is of maximal rank.

The following theorem, which is our main result, is proven in $\S 2$ : 
THEOREM 1. If $\partial f\left(x_{0}\right)$ is of maximal rank, then there exist neighborhoods $U$ and $V$ of $x_{0}$ and $f\left(x_{0}\right)$ respectively, and a Lipschitzian function $g: V \rightarrow R^{n}$ such that

(a) $g(f(u))=u$ for every $u \in U$

(b) $f(g(v))=v$ for every $v \in V$.

When $f$ is $C^{1}, \partial f\left(x_{0}\right)$ reduces to $J f\left(x_{0}\right)$, and the function $g$ above is necessarily $C^{1}$ as well. Thus we recover the classical theorem.

REMARK 1. There are instances of Lipschitz functions which satisfy the conclusions but not the hypotheses of the theorem. Note that it is not enough to assume that $J f$ is of maximal rank whenever it exists, as the function $|x|(n=1)$ demonstrates. A simple example to which the theorem applies $(n=2)$ is the following: $f(x, y)=[|x|+y, 2 x+|y|]$, near $(0,0)$. We find

$$
\partial f(0,0)=\left\{\left[\begin{array}{ll}
s & 1 \\
2 & t
\end{array}\right]:-1 \leqq s \leqq 1,-1 \leqq t \leqq 1\right\}
$$

2. Proof of the theorem. We let $M(0,1)$ denote the unit ball in $\mathcal{M}$.

Lemma 1. Let $\epsilon$ be a positive number. Then for all $x$ sufficiently near $x_{0}$,

$$
\partial f(x) \subset \partial f\left(x_{0}\right)+\epsilon \mathcal{M}(0,1) .
$$

This is a direct consequence of Definition 1, which is equivalent to the following: $\partial f$ is the smallest convex-valued upper-semicontinuous setvalued mapping which contains $J f$ whenever the latter exists.

We suppose that $h: R^{n} \rightarrow R$ is a given $C^{1}$ function, and that $f$ is Lipschitzian near $x$. We denote by $D f$ the derivative of $f$ when it exists.

Lemma 2.

$$
\partial(h \circ f)(x) \subset \nabla h(f(x)) \partial f(x) .
$$

Proof. According to [2, Proposition 1.11], we may express the generalized gradient of the Lipschitzian function $h \circ f$ as in Definition 1 (for $h \circ f$ replacing $f$ ) with the added condition that $x_{t}$ lie in the complement of $E$, where $E$ is any set of measure 0 , without affecting the result. Let us choose for $E$ the set of points (near $x$ ) where $D f$ fails to exist. At any point $y$ not in $E$, by the chain rule we have 


$$
\nabla(h \circ f)(y)=\nabla h(f(y)) J f(y) .
$$

Thus $\partial(h \circ f)(x)$ is the convex hull of a set of points each of which is of the form

$$
\lim _{\imath \rightarrow \infty} \nabla h\left(f\left(y_{\imath}\right)\right) J f\left(y_{\imath}\right)
$$

where $y_{t}$ converges to $x$. But any such point belongs to

$$
\nabla h(f(x)) \partial f(x)
$$

The result now follows from the fact that this last set is convex.

We let $B$ denote the open unit ball in $R^{n}$.

LEMMA 3. There are positive numbers $r$ and $\delta$ with the following property: given any unit vector $v$ in $R^{n}$, there is a unit vector $w$ in $R^{n}$ such that, whenever $x$ lies in $x_{0}+r B$ and $M$ belongs to $\partial f(x)$, then

$$
w \cdot(M v) \geqq \delta .
$$

Proof. (Note that we think of vectors as columns, and that the required vector $w$ is to depend only on $v$, and not on $x$.) Let $S$ denote the unit sphere in $R^{n}$.

The subset $\partial f\left(x_{0}\right) S$ of $R^{n}$ is compact and does not contain 0 , since $\partial f\left(x_{0}\right)$ is of maximal rank. Hence for some $\delta>0, \partial f\left(x_{0}\right) S$ is distance $2 \delta$ from 0 . For positive $\epsilon$ sufficiently small, $G S$ is distance at least $\delta$ from 0 , where $G=\partial f\left(x_{0}\right)+\epsilon M(0,1)$. By Lemma 1 , it follows that for some positive $r$,

$$
x \in x_{0}+r B \Rightarrow \partial f(x) \subset G .
$$

We may suppose $r$ chosen so that $f$ satisfies (1) on $x_{0}+r \bar{B}$.

Now let any unit vector $v$ be given. It follows from the above that the convex set $G v$ is distance at least $\delta$ from 0 . By the usual separation theorem for convex sets, there is a unit vector $w$ such that

$$
w \cdot(\gamma v) \geqq \delta
$$

for every $\gamma$ in G. Relation (2) follows from this along with (3).

Lemma 4. If $x$ and $y$ lie in $x_{0}+r \bar{B}$, then 


$$
|f(x)-f(y)| \geqq \delta|x-y|
$$

Proof. We may suppose $x \neq y$ and (in view of the continuity of $f$ ) $x, y \in x_{0}+r B$. Set

$$
\begin{aligned}
& v=(y-x) /|y-x| \\
& \lambda=|y-x|
\end{aligned}
$$

so that $y=x+\lambda v$.

Let $\pi$ be the plane perpendicular to $v$ and passing through $x$. The set $P$ of points $x^{\prime}$ in $x_{0}+r B$ where $D f(x)$ fails to exist is of measure 0 , and hence by Fubini's theorem, for almost every $x^{\prime}$ in $\pi$, the ray

$$
x^{\prime}+t v, \quad t \geqq 0
$$

meets $P$ in a set of 0 one-dimensional measure. Choose an $x^{\prime}$ with the above property and sufficiently close to $x$ so that $x^{\prime}+t v$ lies in $x_{0}+r B$ for every $t$ in $[0, \lambda]$. Then the function

$$
t \rightarrow f\left(x^{\prime}+t v\right)
$$

is Lipschitzian for $t$ in $[0, \lambda]$ and has a.e. on this interval the derivative $J f\left(x^{\prime}+t v\right) v$. Thus

$$
f\left(x^{\prime}+\lambda v\right)-\left(f\left(x^{\prime}\right)=\int_{0}^{\lambda} J f\left(x^{\prime}+t v\right) v d t\right.
$$

Let $w$ correspond to $v$ as in Lemma 3. We deduce

$$
w \cdot\left[f\left(x^{\prime}+\lambda v\right)-f\left(x^{\prime}\right)\right]=\int_{0}^{\lambda} w \cdot\left[J f\left(x^{\prime}+t v\right) v\right] d t \geqq \int_{0}^{\lambda} \delta d t=\delta \lambda .
$$

Recalling the definition of $\lambda$, we arrive at:

$$
\left|f\left(x^{\prime}+\lambda v\right)-f\left(x^{\prime}\right)\right| \geqq \delta|x-y| .
$$

This may be done for $x^{\prime}$ arbitrarily close to $x$. Since $f$ is continuous, the lemma ensues.

Lemma 5. $f\left(x_{0}+r B\right)$ contains $f\left(x_{0}\right)+(r \delta / 2) B$.

Proof. Let $y$ be any point in $f\left(x_{0}\right)+(r \delta / 2) B$, and let the minimum of $|y-f(\cdot)|^{2}$ over $x_{0}+r \bar{B}$ be attained at $x$. We claim $x$ belongs to $x_{0}+r B$. Otherwise, by Lemma 4 and the triangle inequality, 


$$
\begin{aligned}
r \delta / 2 & >\left|y-f\left(x_{0}\right)\right| \geqq\left|f(x)-f\left(x_{0}\right)\right|-|y-f(x)| \\
& \geqq \delta\left|x-x_{0}\right|-|y-f(x)| \\
& \geqq \delta r-\left|y-f\left(x_{0}\right)\right|>\delta r-\delta r / 2=r \delta / 2,
\end{aligned}
$$

which is a contradiction. Thus $x$ yields a local minimum for the function $|y-f(\cdot)|^{2}$, and consequently (see [2, Corollary 1.10])

$$
0 \in \partial|y-f(x)|^{2}
$$

We now use Lemma 2 to conclude that 0 belongs to the set

$$
2(y-f(x))^{t} \partial f(x)
$$

But Lemma 3 implies that every matrix in $\partial f(x)$ is invertible, hence the above is possible only if $f(x)=y$.

We now set $V=f\left(x_{0}\right)+(r \delta / 2) B$, and we define $g$ on $V$ as follows: $g(v)$ is the unique $x$ in $x_{0}+r B$ such that $f(x)=v$. We choose $U$ as any neighborhood of $x_{0}$ satisfying $f(U) \subset V$. The theorem is now seen to follow, since Lemma 4 implies that $g$ is Lipschitz with constant $1 / \delta$.

REMARK 2. The essential result of Lemma 5 is that the image under $f$ of $x_{0}+r B$ is a neighborhood of $f\left(x_{0}\right)$. H. Halkin and the referee have pointed out that this follows from Lemma 4 and the invariance of domain theorem. We retain our present proof for its elementary nature.

REMARK 3. When $f$ is "strongly differentiable", $\partial f$ reduces to the derivative. Thus Theorem 1 extends the known result in this case [1] [3] (in finite dimensions).

REMARK 4. Following the author's introduction of generalized gradients, two further (and successively more general) extensions of the notion of derivative have been introduced. J. Warga [6] [7] develops the concept of "derivate container"; it can be shown that Theorem 1 is a special case of his "implicit point" theorem. H. Halkin [4] [5] obtains the closely related "interior mapping" theorem (and other results) via the notion of "screen".

REMARK 5. The theorem remains true (with slight modifications in proof) if in Definition 1 we impose the constraint that the points $x_{t}$ lie in the complement of a given set $E$ of measure zero (let us call the resulting generalized Jacobian $\partial_{E} f$ ). Thus the maximality of rank need be verified 
only for $\partial_{E} f\left(x_{0}\right)$ rather than $\partial f\left(x_{0}\right)$. When $f: R^{n} \rightarrow R$ is real-valued, it is known that $\partial_{E} f=\partial f([2$, Proposition 1.11]). It has been pointed out by $\mathrm{H}$. Halkin and J. Warga that the question of whether this is true in the vector-valued case is more profound. We remark that this question seems related to a possible "Denjoy property" for vector derivatives.

\section{REFERENCES}

1. N. Bourbaki, Variétés différentielles et analytiques, Hermann, Paris.

2. F. H. Clarke, Generalized gradients and applications, Trans. Amer. Math. Soc., 205 (1975), 247-262.

3. A. Nijenhuis, Strong derivatives and inverse mappings, Amer. Math. Monthly, 81 (1974), 969-980.

4. H. Halkin, Interior mapping theorem with set valued derivatives, Journal d'Analyse Mathématique, to appear.

5. Mathematical programming without differentiability, Proceedings of "MRC Symposium on the Calculus of Variations and Optimal Control" (September, 1975), D. L. Russell, Ed., Mathematics Research Center, University of Wisconsin, Academic Press, N.Y. (1976).

6. J. Warga, Controllability and necessary conditions in unilateral problems without differentiability assumptions, to appear.

7. - Derivate containers, inverse functions and controllability, Proceedings of "MRC Symposium on the Calculus of Variations and Optimal Control" (September, 1975), D. L. Russell, Ed., Mathematics Research Center, University of Wisconsin, Academic Press, N.Y. (1976).

Received December 3, 1975. Supported in part by National Research Council of Canada grant A 9082.

UNIVERSiTy OF BRItish COLUMBia 



\section{Pacific Journal of Mathematics}

Vol. 64, No. 1

May, 1976

Walter Allegretto, Nonoscillation theory of elliptic equations of order $2 n \ldots \ldots \quad 1$

Bruce Allem Anderson, Sequencings and starters.................. 17

Friedrich-Wilhelm Bauer, A shape theory with singular homology .......... 25

John Kelly Beem, Characterizing Finsler spaces which are

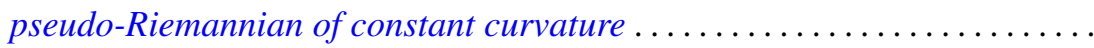

Dennis K. Burke and Ernest A. Michael, On certain point-countable

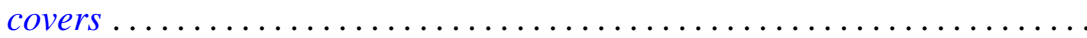

Robert Chen, A generalization of a theorem of Chacon ............... 93

Francis H. Clarke, On the inverse function theorem ................ 97

James Bryan Collier, The dual of a space with the Radon-Nikodým

property ....................................... 103

John E. Cruthirds, Infinite Galois theory for commutative rings ............ 107

Artatrana Dash, Joint essential spectra......................... 119

Robert M. DeVos, Subsequences and rearrangements of sequences in FK

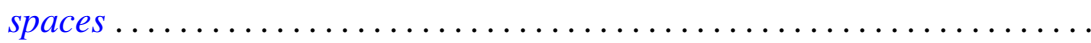

Geoffrey Fox and Pedro Morales, Non-Hausdorff multifunction generalization

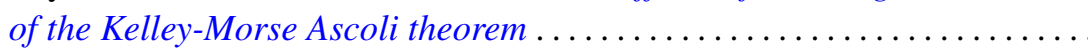

Richard Joseph Fleming, Jerome A. Goldstein and James E. Jamison, One

parameter groups of isometries on certain Banach spaces.............

Robert David Gulliver, II, Finiteness of the ramified set for branched

immersions of surfaces

Kenneth Hardy and István Juhász, Normality and the weak cb property ...... 167

C. A. Hayes, Derivation of the integrals of $L^{(q)}$-functions.

Frederic Timothy Howard, Roots of the Euler polynomials .

Robert Edward Jamison, II, Richard O'Brien and Peter Drummond Taylor, On

embedding a compact convex set into a locally convex topological vector space ....................................

Andrew Lelek, An example of a simple triod with surjective span smaller than span ...

Janet E. Mills, Certain congruences on orthodox semigroups

Donald J. Newman and A. R. Reddy, Rational approximation of $e^{-x}$ on the positive real axis.

John Robert Quine, Jr., Homotopies and intersection sequences ...

Nambury Sitarama Raju, Periodic Jacobi-Perron algorithms and fundamental units ....

Herbert Silverman, Convexity theorems for subclasses of univalent functions. . .

Charles Frederick Wells, Centralizers of transitive semigroup actions and endomorphisms of trees.........................

Volker Wrobel, Spectral approximation theorems in locally convex spaces ..................... 